\title{
Meeting the mental health needs of low- and middle-income countries: the start of a long journey
}

\author{
Steve Kisely and Dan Siskind
}

\section{Summary}

Mental health is increasingly recognised as an important component of global health. In recognition of this fact, the European Union funded the Emerald programme (Emerging Mental Health Systems in Low- and Middle-Income Countries). The aims were to improve mental health in the following six low- and middle-income countries (LMICS): Ethiopia, India, Nepal, Nigeria, South Africa and Uganda. The Emerald programme offers valuable insights into addressing the mental health needs of LMICs. It provides a framework and practical tools. However, it will be important to evaluate longer-term effects including improvements in mental health outcomes, as well as the applicability to LMICs beyond existing participant countries. Importantly, this must be coupled with efforts to improve health worker retention in LMICS.

\section{Declaration of interest}

None.

\section{Keywords}

Low and middle income countries; transcultural psychiatry; clinical governance; education and training.

\section{Copyright and usage}

(C) The Author(s) 2019. This is an Open Access article, distributed under the terms of the Creative Commons AttributionNonCommercial-ShareAlike licence (http://creativecommons. org/licenses/by-nc-sa/4.0/), which permits non-commercial reuse, distribution, and reproduction in any medium, provided the same Creative Commons licence is included and the original work is properly cited. The written permission of Cambridge University Press must be obtained for commercial re-use.
Steve Kisely (pictured) is a psychiatrist, addiction and public health physician who trained in the UK and Australia. He works as a Professor at the University of Queensland and Adjunct Professor in Psychiatry, Community Health and Epidemiology at Dalhousie University in Canada. He was one of the investigators on an international World Health Organization epidemiological study of mental health and physical comorbidity in primary care. Dan Siskind trained as a psychiatrist in Australia and the USA. After working with Médecins Sans Frontières in Chechnya in 2000, he did his psychiatry residency at the Boston University Medical Center and a Master of Public Health at Harvard University. He is a clinical academic psychiatrist at the Metro South Addiction and Mental Health Service in Brisbane

\section{Background}

Mental health is increasingly recognised as an important component of global health. In 2015, the United Nations specifically included the importance of mental health to achieving sustainable development goals (SDGs) in areas such as poverty, hunger and climate change. ${ }^{1}$ For instance, one goal is the reduction of mortality from chronic non-communicable disease by a third through the promotion of mental health and well-being. Other SDGs called for the prevention and treatment of substance use, while those relating to poverty reduction and inequality highlighted the relevance of mental health to many of these issues.

The subsequent Lancet Commission on global mental health and sustainable development further developed these themes. ${ }^{2}$ The Commission highlighted that mental health is a fundamental human right and integral to development in all countries, irrespective of their wealth. In response to these issues, the Commission advocated further investment in mental health services as an essential component of universal health coverage, including more research and innovation, as well as addressing barriers to care and risks to mental health. Importantly, the promotion and protection of mental health is crucial not only in the health sector, but also in the sectors of education, work, social welfare, child and youth services, criminal justice and humanitarian assistance.
If mental health problems are under-recognised and poorly treated in high-income countries, the problems increase by several orders of magnitude in low and middle-income countries (LMICs) where $5 \%$ of people with these disorders receive any treatment or care. ${ }^{3}$ In recognition of this deficit, the European Union (EU) funded the Emerald programme (Emerging Mental Health Systems in Low- and Middle-Income Countries). ${ }^{3}$ The aims were to improve mental health in the following six LMICs: Ethiopia, India, Nepal, Nigeria, South Africa and Uganda. These countries offered a diversity of geographical, economic, social-cultural and urban or rural contexts, as well as a strong commitment of local researchers and policymakers to the programme.

\section{The Emerald programme}

The BJPsych Open Emerald thematic series featured in a recent issue $(2019,5(5))$ are therefore a welcome addition to the literature. Emerald aimed to improve mental health outcomes in the six participating countries by generating evidence, and training professionals and policymakers to enhance health system performance and mental healthcare in these countries. The project first identified barriers to the effective delivery of mental health services, and then offered solutions for improved delivery in the future. There was a strong emphasis on the integration of mental health into primary and maternal health services building on the UK-funded Programme for Improving Mental Health Care (PRIME) in five of the same countries. ${ }^{4}$

In the eight papers of the Emerald thematic series, the programme is introduced and five areas of work described: capacity building, mental health financing, integration of services into primary and community healthcare, the development of mental health information systems with implementation of healthcare indicators and knowledge transfer. ${ }^{3}$ Capacity building encompassed workshops for patients, caregivers, policymakers and researchers 
to raise awareness of advocacy system planning, research capacity and collaboration. ${ }^{5}$ Effects were assessed in terms of $\mathrm{PhD}$ student grant applications and papers as well as questionnaires distributed to participants. ${ }^{5}$

In terms of service delivery and financing models to improve mental healthcare, universal health insurance, along with a greater integration into primary and community care, were the most promising approaches. ${ }^{6}$ These recommendations very much echo those of the Lancet Commission and PRIME. ${ }^{2,4}$ Strengthening the overall health system rather than considering mental health separately may avoid past problems with parallel physical and mental health services. ${ }^{1}$ Other important elements are task sharing, person-centred care and community development beyond just healthcare.

Specifically, the project developed a set of tools to estimate the resources needed to achieve equitable and sustainable mental health financing. ${ }^{6}$ However, the Emerald programme also highlights that interventions must consider wider social determinants such as violence, poverty, unemployment, trauma and stigma that both heighten the risk of mental ill health and the adverse consequences of those who are already ill, including direct financial costs. ${ }^{6,7}$

A further paper in the Emerald thematic series used interviews from managers and service providers to highlight that successful integration required more than the training of existing primary care clinicians. There was a need for systemic structural change including a greater overall emphasis on the management of chronic disease of all forms with support from specialists and improved information systems. ${ }^{8}$ Examples included intervention guidelines, care pathways and better access to psychotropic medication.

Using a two-round Delphi process of 93 experts, Emerald project researchers developed a set of key indicators for mental health programme monitoring in primary care. ${ }^{9,10}$ In the first round, participants completed a survey to generate candidate indicators. ${ }^{9}$ In the second, the 15 highest scoring indicators on a five-point Likert scale were selected based on significance, relevance and feasibility. ${ }^{9}$ These key indicators covered contacts for mental health problems, unmet needs of people with mental health problems, the quality of services provided and the associated financial risk to the person and their family. Their utility was assessed in a survey of primary healthcare workers, as well as the completeness and accuracy of data completion in randomly selected health records. ${ }^{10}$ Barriers to collection were similar to those elsewhere, including time pressure and overstretched health workers, although this was mitigated by simplicity of the forms and commitment of staff. ${ }^{11}$

\section{Generalisability}

The Emerald programme suggests useful and practical steps to addressing the major unmet needs in LMICs identified by the Lancet Commission. Nevertheless, there are some limitations. Countries were partly selected based on the commitment and enthusiasm of local services. Findings may therefore be less applicable to countries, or even other parts of the participating nations, where services are less committed, organised or even absent. Given the programme was funded by the EU, it is notable that there were no countries from the Caribbean, Oceania, Latin America or francophone Africa, which may again limit generalisability. Integration with primary and community care is stressed but there is little mention of traditional healers who play an important role in healthcare. For instance, a recent review of 40 studies on schizophrenia from eight countries in Sub-Saharan Africa found that most people were treated by both mainstream psychiatry and faith or traditional healers. ${ }^{12}$

Much of the programme evaluation was short term and based on quantitative and qualitative assessment of process or proxy measures rather than mental health outcomes. To some extent, this was unavoidable given the time constraints of the Emerald programme, but may again limit generalisability. ${ }^{13}$ Although potentially useful tools were developed, it is unclear to what degree they will be adopted.

\section{Child maltreatment}

Strengthening of healthcare services is only one component to improving mental health. As highlighted in the concluding paper of the BJPsych Open Emerald thematic series, there is also a need to 'upstream' determinants of mental health such as violence, poverty, poor housing, unemployment, limited educational opportunities and past experiences of trauma and stigma. ${ }^{7}$ Not mentioned specifically are the long-term effects of child maltreatment on mental health. ${ }^{1}$ These include physical, emotional and sexual abuse, as well as neglect. ${ }^{14}$ Globally, substantial numbers of children have experienced childhood maltreatment, although prevalence may vary in terms of timing and type, as well as across countries over time. ${ }^{15}$ Thus, estimates of the population prevalence of childhood maltreatment range from $12 \%$ for sexual abuse to $36.3 \%$ for emotional abuse. ${ }^{15}$

\section{Human rights issues}

It is also important to consider human rights issues, including specifically those around compulsory psychiatric treatment. ${ }^{16}$ For instance, people with severe mental illness may experience major abuses including forced restraints, violence and torture. ${ }^{2}$ Even where safeguards may exist, the compliance of mental health legislation with human rights instruments is poor in low-income countries. $^{16}$

\section{Emigration of healthcare professionals}

Even in the presence of compliant mental health legislation, there are often insufficient specialists to provide assessments or reviews. ${ }^{16}$ Related to this problem, is the emigration of medical and nursing professionals to wealthier countries. The availability of trained personnel is not just dependent on capacity building but also retaining existing talent. Central to this is countering the 'brain drain' of mental health clinicians from LMICs to wealthier countries. ${ }^{17}$ For instance, a third of doctors and $50 \%$ of nurses registered to practice in the UK are from overseas. ${ }^{17}$ The biggest group of overseas doctors came from either India or Pakistan. In Australia, $52 \%$ of doctors who arrived on permanent or temporary visas in the 5 years from 2005 were from India, Malaysia, China, The Philippines, South Africa or Egypt. ${ }^{18}$ There are similar proportions of international medical graduates in North America, with more than half coming from LMICs. ${ }^{19}$

\section{Conclusions}

In conclusion, the Emerald programme offers valuable insights into addressing the mental health needs of LMICs. It provides a framework and practical tools. It will be important to evaluate longerterm effects including improvements in mental health outcomes, 
as well as applicability to LMICs beyond existing participant countries. Importantly, this must be coupled with efforts to improve health worker retention in LMICs.

Steve Kisely $\mathbb{D}, \mathrm{MD}, \mathrm{PhD}$, DMedRes, Professor, School of Medicine, University of Queensland, Princess Alexandra Hospital; Metro South Addiction and

Mental Health Service, Australia; and Adjunct Professor, Departments of Psychiatry,

Community Health and Epidemiology, Dalhousie University, Canada; Dan Siskind (D)

MBBS, MPH, PhD, Associate Professor, School of Medicine, University of Queensland,

Princess Alexandra Hospital; and Metro South Addiction and Mental Health Service,

Brisbane, Australia

Correspondence: Steve Kisely. Email: s.kisely@uq.edu.au

First received 2 Sep 2019, final revision 17 Oct 2019, accepted 18 Oct 2019

\section{References}

1. Jenkins R. Global mental health and sustainable development 2018. BJPsych Int 2019; 16: 34-7.

2 Patel V, Saxena S, Lund C, Thornicroft G, Baingana F, Bolton P, et al. The Lancet Commission on global mental health and sustainable development. Lancet 2018; 392: 1553-98.

3 Thornicroft G, Semrau M. Health system strengthening for mental health in lowand middle-income countries: introduction to the Emerald programme. BJPsych Open 2019; 5: e66.

4 Breuer E, Hanlon C, Bhana A, Chisholm D, De Silva M, Fekadu A, et at. Partnerships in a global mental health research programme-the example of PRIME. Global Soc Welfare 2019; 6: 159-75.

5 Evans-Lacko S, Hanlon C, Alem A, Ayuso-Mateos J, Chisholm D, Gureje O, et al. Evaluation of capacity-building strategies for mental health system strengthening in low- and middle-income countries for service users and caregivers, policymakers and planners, and researchers. BJPsych Open 2019; 5: e67.

6 Chisholm D, Docrat S, Abdulmalik J, Alem A, Gureje O, Gurung D, et al. Menta health financing challenges, opportunities and strategies in low- and middleincome countries: findings from the Emerald project. BJPsych Open 2019; 5: e68.
7 Semrau M, Alem A, Ayuso-Mateos J, Chisholm D, Gureje O, Hanlon C, et al. Strengthening mental health systems in low- and middle-income countries: recommendations from the Emerald programme. BJPsych Open 2019; 5: e73.

8 Petersen I, Van Rensburg A, Kigozi F, Semrau M, Hanlon C, Abdulmalik J, et al. Scaling up integrated primary mental health in six low- and middle-income countries: obstacles, synergies and implications for systems reform. BJPsych Open 2019; 5: e69.

9 Jordans MJ, Chisholm D, Semrau M, Upadhaya N, Abdulmalik J, Ahuja S, et al. Indicators for routine monitoring of effective mental healthcare coverage in low- and middle-income settings: a Delphi study. Health Policy Plann 2016; 31: 1100-6.

10 Jordans M, Chisholm D, Semrau M, Gurung D, Abdulmalik J, Ahuja S, et al. Evaluation of performance and perceived utility of mental healthcare indicators in routine health information systems in five low- and middle-income countries. BJPsych Open 2019; 5: e70.

11 Ahuja S, Hanlon C, Chisholm D, Semrau M, Gurung D, Abdulmalik J, et al. Experience of implementing new mental health indicators within information systems in six low- and middle-income countries. BJPsych Open 2019; 5: e71.

12 Chidarikire S, Cross M, Skinner I, Cleary M. Treatments for people living with schizophrenia in Sub-Saharan Africa: an adapted realist review. Int Nurs Rev 2018; 65: 78-92.

13 Ayuso-Mateos J, Miret M, Lopez-Garcia P, Alem A, Chisholm D, Gureje O, et al. Effective methods for knowledge transfer to strengthen mental health systems in low- and middle-income countries. BJPsych Open 2019; 5: e72.

14 Kisely S, Abajobir AA, Mills R, Strathearn L, Clavarino A, Najman JM. Child maltreatment and mental health problems in adulthood: birth cohort study. Br J Psychiatry 2018; 213: 698-703.

15 Stoltenborgh M, Bakermans-Kranenburg MJ, Alink LR, van Ijzendoorn MH. The prevalence of child maltreatment across the globe: review of a series of metaanalyses. Child Abuse Rev 2015; 24: 37-50.

16 Eaton J. Human rights-based approaches to mental health legislation and global mental health. BJPsych Int 2019; 16: 37-40.

17 Jenkins R. Brain drain. Int Psychiatry 2016; 13: 53-5.

18 Hawthorne L. International medical migration-what is the future for Australia? MJA Open 2012; 1: 18-21.

19 Oladeji B, Gureje O. Brain drain: a challenge to global mental health. BJPsych Int 2016; 13: 61-3. 